Improving Image Quality and Reducing

Drift Problems via Automated Data

Acquisition and Averaging in

Cs-corrected TEM

E. Voelkl, B. Jiang, Z. R. Dai, J. P. Bradley

September 3, 2008

Microscopy and Microanalysis 
This document was prepared as an account of work sponsored by an agency of the United States government. Neither the United States government nor Lawrence Livermore National Security, LLC, nor any of their employees makes any warranty, expressed or implied, or assumes any legal liability or responsibility for the accuracy, completeness, or usefulness of any information, apparatus, product, or process disclosed, or represents that its use would not infringe privately owned rights. Reference herein to any specific commercial product, process, or service by trade name, trademark, manufacturer, or otherwise does not necessarily constitute or imply its endorsement, recommendation, or favoring by the United States government or Lawrence Livermore National Security, LLC. The views and opinions of authors expressed herein do not necessarily state or reflect those of the United States government or Lawrence Livermore National Security, LLC, and shall not be used for advertising or product endorsement purposes. 


\title{
Improving Image Quality and Reducing Drift Problems via Automated Data Acquisition and Averaging in Cs-corrected TEM
}

\author{
E. Voelkl, ${ }^{1}$ B. Jiang, ${ }^{1,2}$ Z.R. Dai, ${ }^{2}$ and J.P Bradley ${ }^{2}$ \\ ${ }^{1}$ FEI Company, 5350 NE Dawson Creek Drive, Hillsboro, OR 97124, USA \\ 2 Institute of Geophysics and Planetary Physics, Lawrence Livermore National \\ Laboratory, Livermore, CA 94551, USA
}

\section{Introduction}

Image acquisition with a CCD camera is a single-press-button activity: after selecting exposure time and adjusting illumination, a button is pressed and the acquired image is perceived as the final, unmodified proof of what was seen in the microscope. Thus it is generally assumed that the image processing steps of e.g., "dark-current correction" and "gain normalization" do not alter the information content of the image, but rather eliminate unwanted artifacts.

Image quality therefore is, among a long list of other parameters, defined by the dynamic range of the CCD camera as well as the maximum allowable exposure time depending on sample drift (ignoring sample damage). Despite the fact that most microscopists are satisfied with present, standard image quality we found that it is a relatively easy to improve on existing routines in at least two aspects [1]:

1. Suppression of lateral image drift during acquisition by using significantly shorter exposure times with a plurality of exposures (3D-data set).

2. Improvement in the Signal/Noise ratio by averaging over a given data set by exceeding the dynamic range of the camera.

\section{Background}

Images recorded in an electron microscope are usually band-width limited. This can be verified easily by taking a Fourier transform (FFT) of a given image and checking that no structures contribute visibly at the outer edges of the Fourier transform (also known as the Nyquist limit [2]). Otherwise, the image could contain artifacts and should be discarded if in doubt. For the following, it will be assumed that the acquired images are of reasonable quality and thus inherently bandwidth limited at the Nyquist limit. As a reminder, a digital image consists of $M \times N$, usually quadratic, pixels with distance $d$ between consecutive pixels in $x$ - and $y$ - direction. Most importantly: each pixel represents a point and not an image area! This little detail sometimes is conveniently overlooked (mostly without causing any damage), but should be remembered when the relation between the image intensity $I(x, y)$ as a function of $x$ - and $y$-coordinates, versus the pixel values $P_{m, n}$ that represent the intensity function at selected points with coordinates $(m, n)$ needs to be considered. 
The latter could, in principle, be arranged in reality by using a camera with a very small active area (but the resulting detection quantum efficiency (DQE) would become prohibitively low requiring extremely long exposure times).

At this point we are set up for using Shannon's sampling theorem [2]. Simply formulated it states that: if all spatial frequencies that contribute to $I(x, y)$ within the camera area are sampled below the Nyquist limit, then the function $I(x, y)$ is uniquely defined for this area.

This means, that by recording a decent image, we not only know the image intensity at the points $P_{m, n}$, but at any point within the area of the CCD camera according to Shannon's law (and the requirements already discussed). Once it is clear how to obtain the $\mathrm{P}_{m+a, n+b}$ from the $\mathrm{P}_{m, n}$ (with $a$ and $b$ as real numbers) it will be possible to align images within a fraction of a pixel accurately, if the lateral offset between consecutive images is known.

\section{Determining Shift Between Consecutive Images}

The standard routine for determining a shift (or lateral offset) between images is the cross-correlation function. The literature on this issue is overwhelming and plenty of good scholarly articles can be found directly on the Internet. In many cases, additional filters (e.g., Apodization windows) are being used to reduce potential artifacts and increase the accuracy of the method. It may be sufficient to point out here that for the specific purpose of this paper images are expected to shift within reason (i.e., significantly less than $1 / 4$ of the image area) and not change during the total acquisition time frame (i.e., no sample damage or defocus). Under these circumstances, the crosscorrelation function yields generally excellent and reliable results and these functions are implemented in HoloWorks transparently and normally don't require user interaction.

\section{Shifting Images With sub-Pixel Accuracy}

This question was answered essentially in [3] and the reader inclined to review the mathematics is recommended to read these articles. However, there it was only shown how to shift a Fourier transform by an arbitrary amount. Luckily, the mathematics for shifting a Fourier transform or shifting an image in real space is essentially the same. For a basic understanding it may be sufficient to realize that if the FFT of an image (which has complex value data) is multiplied by a phase wedge in $x$ - direction $(\cos (m \varphi / M)$, i $\sin (m \varphi / M))$, the resulting inverse FFT will provide an image that is shifted in real space. For example, if the phase wedge is in $x$ - direction only and the slope is linear and increases from 0 to $2 \pi$ from left to right $(\varphi=2 \pi)$, then the resulting image shift in real space is exactly 1 pixel (within computational accuracies). Obviously, fractions of pixel shifts are obtained by fractions of a $2 \pi$. Therefore, correcting a known image shift entails 2 Fourier transforms and one multiplication. This is a rapid process and usually takes significantly less than $1 \mathrm{~s}$ per image. 


\section{Experimental Set-up}

The experiment was performed on a Titan with an image $\mathrm{C}_{\mathrm{S}}$ corrector at $300 \mathrm{kV}$. On these instruments specifically, the illumination system is extremely stable and changes in the illuminating wave front (especially its phase) are smaller by at least an order of magnitude compared to conventional TEMs [4]. The sample was a standard Au on carbon film specimen, referred to as cross-grating. For image acquisition and processing, standard routines of DigitalMicrograph [5] and HoloWorks [6] were used.

First, the camera was started in its "continuous" acquisition mode with a binning factor of 2 and an exposure time $t$ of $1 \mathrm{~s}$. The magnification was chosen carefully to avoid aliasing while recording the largest possible field of view. Therefore, the bandwidth limit of the acquired images is close to the Nyquist limit but does not exceed it. Using HoloWorks' $3 \mathrm{D}$ acquisition routine, 50 images were acquired within a 3D data stack (containing 50 "slices") thus avoiding cluttering of the screen with 50 individual images. Once the image acquisition was complete, the averaging routine (provided also by HoloWorks) was started and within a minute returned details about 1) the offset between images, 2) an aligned stack where all slices are aligned laterally and 3) the averaged, noise-improved image.

Since the cumulative data acquisition "piggybacks" onto the continuous data acquisition of DigitalMicrograph camera independence is assured, at least within the framework of

DM. As a precaution, the aligned 3D-data stack was inspected visually at a zoom factor of 4 for verification of the alignment process and no problems were found. The data were acquired with a binning factor of 2 .

\section{Results}

In FIG. 1, the results from the experiment are displayed: the contrast range for the high resolution images (all images on the left) is based on their respective histogram for a decent contrast range selection - i.e., the default setting within DM. The contrast range for the Fourier images however was fixed for all images on the right-hand side for better comparison.

At the top of Fig. 1, the first of the 50 images (to the right its corresponding Fourier image) is displayed. A slight astigmatism is visible in the Fourier image. In the middle, the result from simply averaging all 50 images without alignment is displayed. Because the sample was drifting at an average rate of $0.028 \mathrm{~nm} / \mathrm{s}$, (see data in Fig. 2) the typical effect of smearing is visible and the corresponding Fourier image (on the right) confirms the loss of information in the main drift direction.

At the bottom of Fig. 1, the result from averaging the 50 images after a successful lateral alignment is displayed. Its corresponding Fourier image displays a significantly higher quality than the Fourier image of just one slice. Especially the shape function of each crystal which is convoluted around each of the peaks in the Fourier image has become much clearer and high-frequency noise is greatly suppressed in general. 


\section{Application of the method}

Having a simple and straight forward method at hand for acquiring optimum high resolution images despite drift is certainly a convenience factor for any TEM laboratory and can result in significant time savings. In addition, quantitative information about sample drift is helpful when deciding what to do. For example, if it is clear that the specimen drift is slowing down, a short wait might be preferred over averaging. If the drift shows random behavior, averaging might be the only solution. As a rule of thumb, drift should not exceed $1 / 4$ of the desired resolution. And having a simple process at hand for acquiring, aligning and averaging e.g., 10 images, this essentially reduces the specimen stability requirements by a factor of 10 .

\section{Further Possibilities}

Given the rather stable illumination wave front of e.g., a Titan microscope [4], the signal/noise ratio of high resolution images can be improved drastically beyond what is possible from a single shot limited by the dynamic range of CCD cameras. As the signal/noise ratio improves in the image, it also improves in the Fourier images. Under these circumstances, the shape factors of crystals play an increasing role and crystals with sharp edges and/or sides that are parallel to the electron beam create so-called side-lobes for the diffracted beams: the Fourier transform of the shape function is convoluted around the diffracted beams.

In Figure 3, left, the peak corresponding to a (-111) beam for a Au crystal in [110] direction as obtained from the averaged image in Figure 1, bottom right, is displayed. Clearly the peak is convoluted with the shape function of the crystal creating that peak and after isolating the peak with an aperture and an inverse Fourier transform the crystal forming the peak is visible and displayed in Figure 3, bottom right. For orientation, the corresponding area from the original image is inserted just above. A thin, dark black line across the crystal is clearly visible. By measuring the width of the line using a linescan displayed in Figure 4, the width of the line is found to correspond to a lattice spacing of $\sim 0.23 \mathrm{~nm}$ which supports the assumption that one atomic layer of the Au crystal is astray.

The high contrast $(\sim 10: 1)$ for the dark line in Figure 4 can only be obtained with a $\mathrm{C}_{\mathrm{S}}$ corrected microscope, because the point spread function caused by the aberrations is sufficiently small to not eradicate this line. Also contributing to the shape of the intensity profile is the size of the aperture used to isolate the peak which can become an issue if the aperture has to be significantly smaller to isolate a certain peak. 


\section{Known limitations:}

Since data sets are limited by the amount of available RAM, the upper limit for the number of images to be stored will differ for different systems. To find the upper limit for a given system is simple, as in case of insufficient memory a simple message "Not enough memory" occurs without causing any further trouble. Sample drift in $z$ - direction (perpendicular to image plane) unfortunately cannot be corrected by this method nor can changes of crystal orientation or any other changes to the sample under the electron beam.

\section{Conclusion}

Due to sample drift problem during TEM image acquisition, image quality can not be improved by extended exposure time. Our experimental showed that image quality can be improved by drift correction via automated data acquisition and averaging. This method was successfully demonstrated on a $\mathrm{C}_{\mathrm{S}}$ corrected TEM. Given further improvements in signal/noise ratio it may be possible to not only relate the diffraction spots to a certain crystal, but also to obtain information about its 3D geometry.

\section{References}

[1] E Voelkl, B Jiang, ZR Dai, and JP Bradley, Microsc Microanal 14(Suppl 2), 2008, 844-845.

[2] MB Priestly (1981) Spectral Analysis and Time Series, Academic Press

[3] E Voelkl et al., Ultramicroscopy 58 (1995) 97-103

[4] E Voelk1, Microsc Microanal 14(Suppl 2), 2008, 840-841.

[5] DigitalMicrograph, software by Gatan, Inc., 5794 W. Las Positas Blvd. Pleasanton, CA

[6] HoloWorks v4, software plug-in for DigitalMicrograph, Gatan Inc., Pleasanton, CA

[7] This research was supported by a NASA Sample Return Laboratory instrument

Development Program (SRLIDAP) grant (to JPB) and a Laboratory Directed Research and Development (LDRD) award, and it was performed under the auspices of the U.S. DOE by Lawrence Livermore National Laboratory in part under contract No. W-7405Eng-48 and in part under contract DE-AC52-07NA27344. 


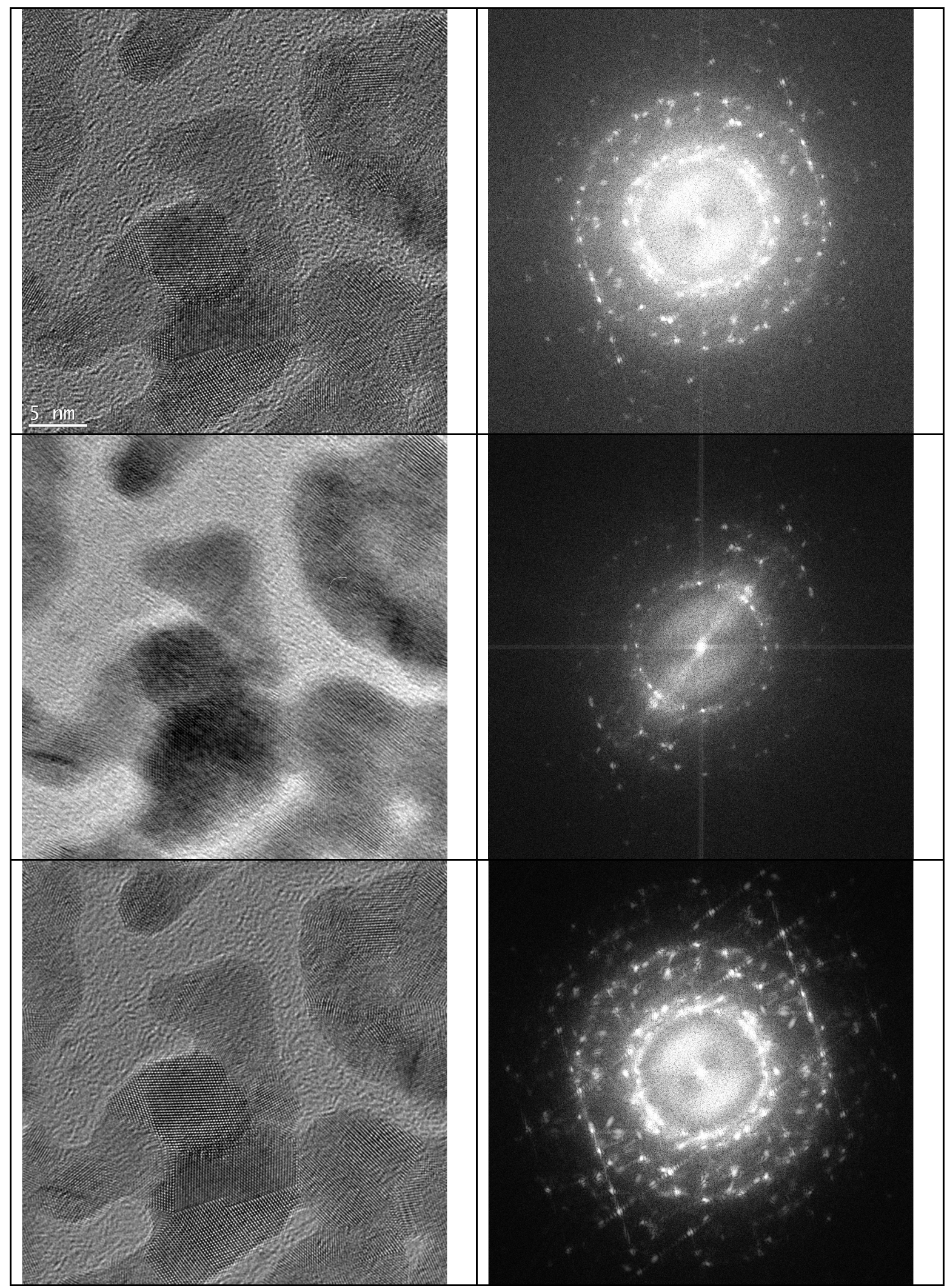

Figure 1 A data set of 50 consecutively images; corresponding Fourier images to the right. Top: one image only. Middle: 50 images averaged. Bottom: all 50 images aligned, then averaged. 


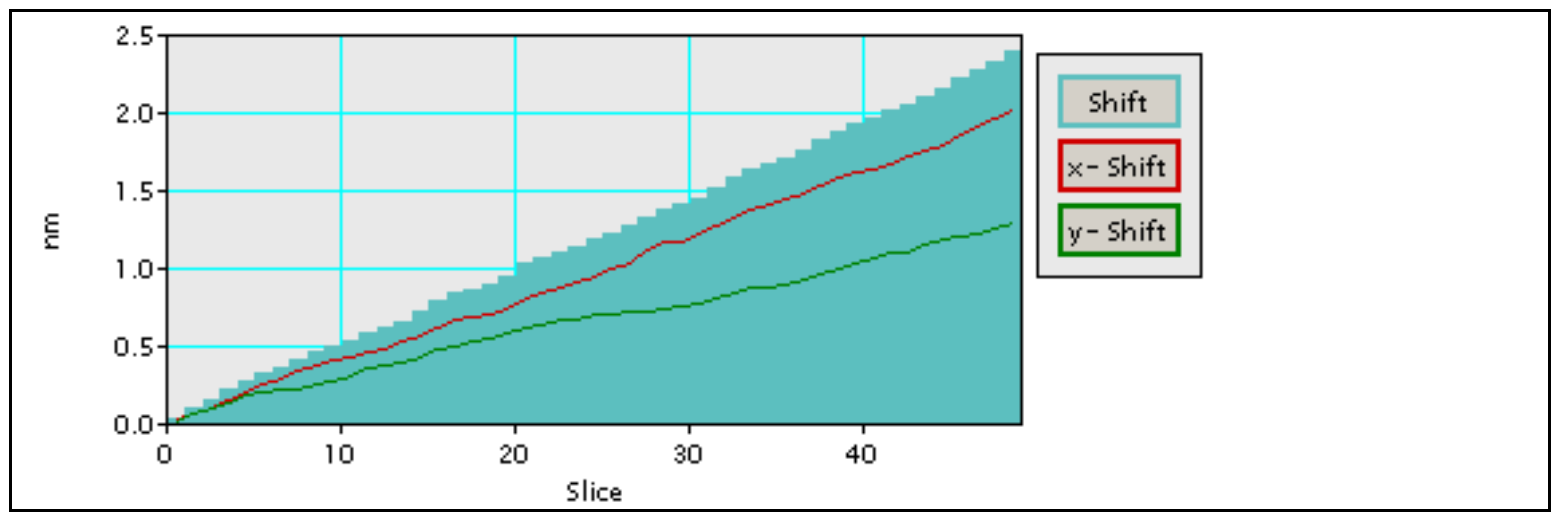

Figure 2 Sample drift as determined from 50 consecutive images. Including overhead for readout time (40s) and an exposure time of $1 \mathrm{~s}$ per image, the average drift computes as $\sim 2.5 \mathrm{~nm} / 90 \mathrm{~s}=$ $0.028 n m / s$.
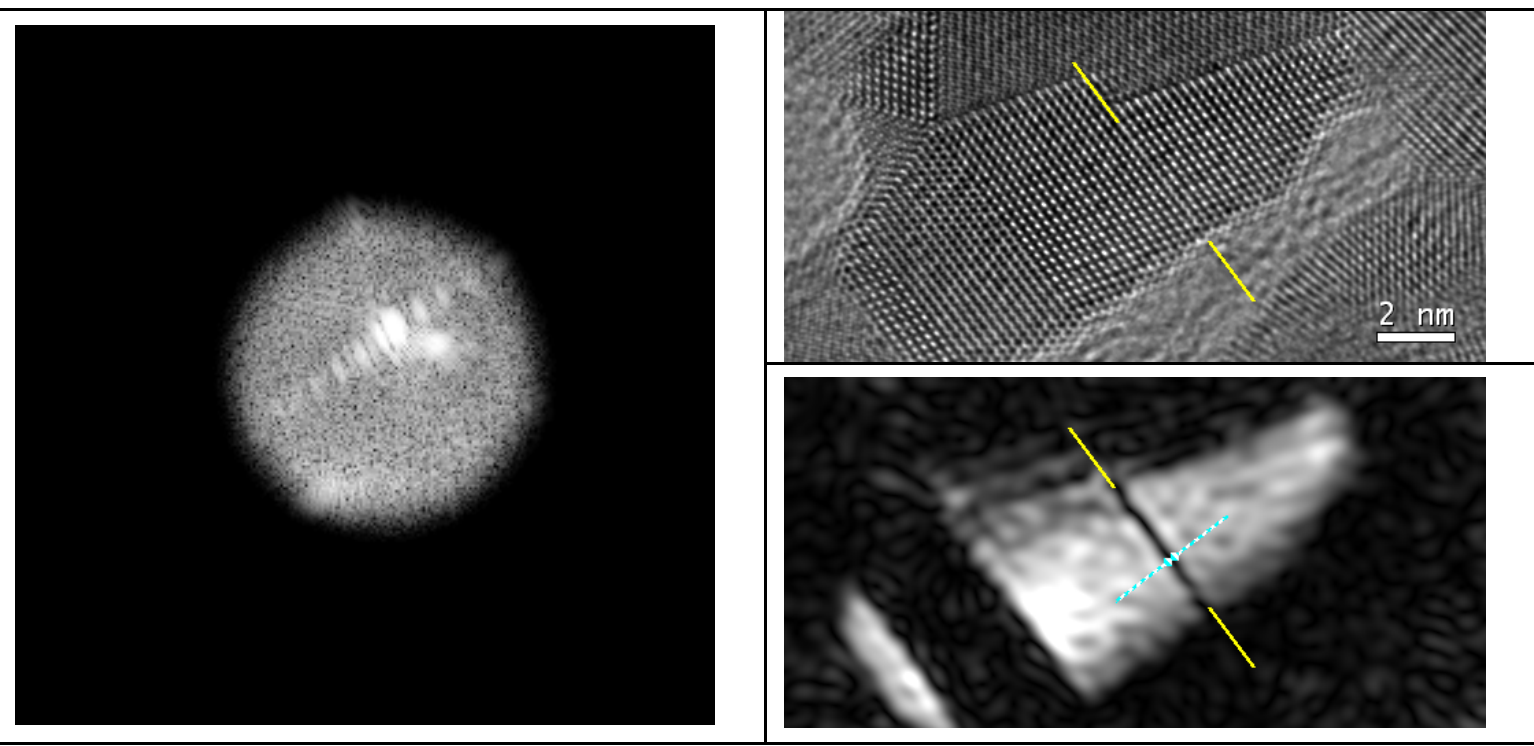

Figure 3 Left: Isolating a (-111) peak with a soft aperture. Right bottom: the Au crystal oriented in [110] has a single atomic layer splitting the crystal in half. Right top: same area in unfiltered image.

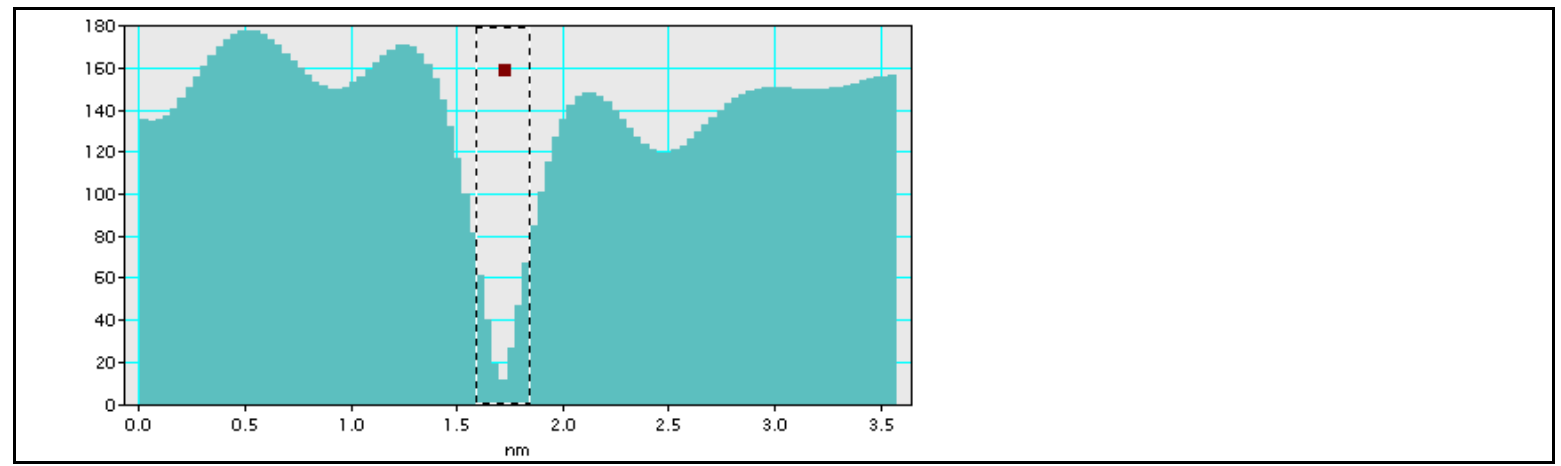

Figure 4 A linescan across the single atom plane in Figure 3, bottom right, that splits two crystals with the same orientation. Due to $\mathrm{C}_{S}$ correction, the contrast for the single atom plane is high. Without $\mathrm{C}_{\mathrm{S}}$ correction, the resulting point spread function would smear most of the contrast. 